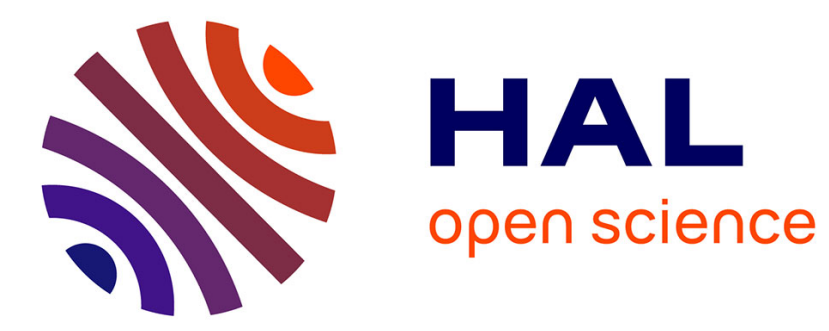

\title{
On scheduling models for the frequency interval assignment problem with cumulative interferences
}

Kata Kiatmanaroj, Christian Artigues, Laurent Houssin

\section{To cite this version:}

Kata Kiatmanaroj, Christian Artigues, Laurent Houssin. On scheduling models for the frequency interval assignment problem with cumulative interferences. Engineering Optimization, 2016, 48 (5), pp.740-755. 10.1080/0305215X.2015.1056789 . hal-01274969

\section{HAL Id: hal-01274969 \\ https://hal.science/hal-01274969}

Submitted on 16 Feb 2016

HAL is a multi-disciplinary open access archive for the deposit and dissemination of scientific research documents, whether they are published or not. The documents may come from teaching and research institutions in France or abroad, or from public or private research centers.
L'archive ouverte pluridisciplinaire HAL, est destinée au dépôt et à la diffusion de documents scientifiques de niveau recherche, publiés ou non, émanant des établissements d'enseignement et de recherche français ou étrangers, des laboratoires publics ou privés. 


\title{
On scheduling models for the frequency interval assignment problem with cumulative interferences
}

\author{
Kata Kiatmanaroj ${ }^{a b}$ and Christian Artigues ${ }^{a b}$ and Laurent Houssin ${ }^{a c *}$ \\ ${ }^{a}$ CNRS, LAAS, 7 avenue du colonel Roche, F-31400 Toulouse, France; ${ }^{b}$ Univ de Toulouse, \\ LAAS, F-31400 Toulouse, France; ${ }^{c}$ Univ de Toulouse, UPS, LAAS, F-31400 Toulouse, France \\ (v4.1 released April 2013)
}

\begin{abstract}
In this paper, models and methods for solving a real-life frequency assignment problem based on scheduling theory are investigated. A realistic frequency assignment problem involving cumulative interference constraints in which the aim is to maximize the number of assigned users is considered. If interferences are assumed to be binary, a multiple carrier frequency assignment problem can be treated as a disjunctive scheduling problem since a user who requests a number of contiguous frequencies can be considered as a non-preemptive task with a processing time and two interfering users can be modeled through a disjunctive constraint on the corresponding tasks. A binary interference version of the problem is constructed and derive a disjunctive scheduling model is derived. Based on the binary representation, two models are proposed. The first one relies on an interference matrix and the second one considers maximal cliques. A third, cumulative, model that yields a new class of scheduling problems is also proposed. Computational experiments show that the case-study frequency assignment problem can be solved efficiently with disjunctive scheduling techniques.
\end{abstract}

Keywords: Frequency assignment; Scheduling; Cumulative interferences; Disjunctive graphs; Maximal cliques

\section{Introduction and related work}

Frequency assignment problem (FAP) first appeared in the work by Metzger (1970) and has been associated with graph coloring problems ever since. The problem is still of great interest today as one observes a continuing increase in wireless communication demands and applications. To deal with this class of difficult (mostly strongly NP-hard) combinatorial optimization problems, a wide variety of models and solving methodologies have been proposed Aardal et al. (2007). Part of the research concentrated on exact methods that can provide an optimal solution to the problem, among which integer linear programming and constraint programming approaches. Heuristics and metaheuristics methods were also considered as they can provide good solutions to the problem in a reasonable amount of time. Recent examples of successful (meta)heuristics for the FAP can be found in Muñoz and Muñoz (2012); Montemanni and Smith (2010).

Among the various FAP models, this paper considers a particular model extracted from practical problems provided by Thales Alenia Space, a French aerospace company (see Kiatmanaroj et al. (2012) for a description of the complete industrial application). Given a set of users, a set of binary interference constraints and a bandwidth represented by a set of integers, the basic FAP consists in assigning a single frequency to each 
user so that no interfering users share the same frequency. Considering the graph where nodes represent users and edges represent the binary interference constraints, the basic FAP is equivalent to the vertex graph coloring problem. The considered model has two additional features. First, each user may request several frequencies instead of a single one (multiple carrier FAP) and these frequencies must be contiguous. It follows that a frequency interval must be assign to each user. Binary interferences indicate that the intervals assigned to two interfering users shall not overlap. The frequency interval assignment problem (FIAP) is equivalent to the interval coloring problem as considered in de Werra and Gay (1994); Bouchard et al. (2010). The second additional characteristic considered lies in the presence of cumulative interferences, i.e. interferences coming from multiple sources. When a single frequency has to be assigned to each user, the cumulative interference model considers a penalty if the same frequency is assigned to a user and one of its neighbors. The interference level becomes unacceptable for a user if the sum of all penalties exceeds a threshold corresponding to the required level of service for this user. Such cumulative or multiple sources interferences, that break the correspondence between FAP and graph coloring, have previously been considered in the literature in Mannino and Sassano (2003); Houssin et al. (2011); K.Kiatmanaroj et al. (2013); Palpant et al. (2008), among others. However, in the interference model considered in this paper, the interference level for a user depends on the cumulated overlapping lengths of its assigned frequency interval with those of its neighbors. Such an interference model, linked to the conjunction of frequency interval assignment and cumulative interferences, was not previously considered in the FAP literature. As providing each user with a frequency interval given a fixed bandwidth may be infeasible, two alternative objectives are considered in this paper. Either the method is to maximize the number of assigned users or to minimize the used bandwidth.

To solve this practical problem, the link between the FIAP and the disjunctive scheduling problem is exploited. According to Pinedo (2012), scheduling is a decision making process that deals with the allocation of resources to tasks over given time periods while the resources and tasks can take many different forms, as well as objectives. By this definition, scheduling is a large and broad subject and has many industrial applications. Nonetheless, in paper de Werra and Gay (1994), a link between chromatic scheduling, graph coloring, and the FAP has been established. Namely, if a user is treated as a task and the user demand (in a number of contiguous frequencies) as the task's processing time, maximizing the number of users assigned with contiguous frequencies can be viewed as maximizing the number of scheduled tasks having their common deadlines set to the number of available frequencies. Similarly, minimizing the used bandwidth amounts to minimize the makespan. When binary interferences are considered, the FIAP is equivalent to the disjunctive scheduling problem with the mentioned objectives. In the presence of deadlines and/or for the makespan criterion, disjunctive scheduling problems are especially well-solved by specialized constraint programming techniques under the constraint-based scheduling framework Dorndorf et al. (2000); Baptiste et al. (2001). However, the cumulative interference constraints yield a new type of limited overlapping scheduling constraints that was not considered before but that can still be easily modeled via constraint programming. In the absence of specialized solving techniques for this new scheduling problem, disjunctive scheduling approximate models are proposed in this paper . Then the direct limited overlapping scheduling model is compared experimentally with the disjunctive approximations on the provided realistic cumulative FIAP instances.

The paper is organised as follows: Section 2 provides a description of the telecommunication system from which the FIAP is issued and gives the expression of the cumulative interference constraints. In Section 3, a limited overlapping scheduling model and a family of disjunctive scheduling approximations for the FIAP are provided. Section 4 presents the experimental results while conclusions are given in Section 5. 


\section{Cumulative interferences in frequency interval assignment}

This paper considers a frequency assignment in a satellite communication system which consists of a satellite, a gateway and a number $n$ of user terminals. The satellite acts as a relay point between the users and the gateway providing bi-directional communication links between the two parties. The gateway, which is excluded in this study, is a communication node that connects the satellite system to the terrestrial network.

User terminals are ground-based and can be referred to as the Earth stations. They are randomly generated and randomly positioned within a fictitious rectangular service area defined by a set of geographic coordinates. The satellite is equipped with antennas or antenna array that can provide a number of satellite beams, each beam centered to each user.

Each user $i \in\{1, \ldots, n\}$ is associated with a demand $d_{i}$ with which the system tries to accommodate by assigning a block of contiguous frequencies $f_{i}, f_{i}+1, \ldots, f_{i}+d_{i}-1$ depending on the size of the demand $d_{i}$. There are a limited number $B W$ of frequencies that the system can assign; nonetheless, assigning the same frequency to other users (i.e. frequency reuse) can be performed providing that this will not cause excessive frequency interference to the users.

It is assumed that there is no adjacent channel interference between frequency pairs $(f, f+1)$ since a frequency separation is required between $f$ and $f+1$. According to this, the problem is to deal with co-channel interferences possibly occurring for two users $i$ and $j, i \neq j$ if $\left[f_{i}, f_{i}+d_{i}-1\right] \cap\left[f_{j}, f_{j}+d_{j}-1\right] \neq \emptyset$. Co-channel interference could occur if the same frequency is used and the users are geographically close to each other. Interference is cumulative and the system can tolerate this interference if the cumulative level does not exceed a predefined threshold.

Link budget which accounts all of the gains and losses from the transmitter to the receiver is determined for each user. Evaluation of the quality of the reception can be done by verifying the signal to noise ratio which is defined as the ratio of the desired signal power to the noise (unwanted signal) power. The noise can be viewed as a combination of thermal noise and interference. This signal to noise ratio is represented as $\left(\frac{C}{N+I}\right)$.

For a successful reception, this $\left(\frac{C}{N+I}\right)$ should not be less than a given value, a required signal to noise ratio, denoted by $\left(\frac{C}{N}\right)_{\text {Required }}$ i.e.

$$
\frac{C}{N+I} \geq\left(\frac{C}{N}\right)_{\text {Required }}
$$

For a typical user $i \in\{1, \ldots, n\}$, the signal to noise and interference ratio is given by Corbel (juillet 2008)

$$
\begin{aligned}
\left(\frac{C}{N+I}\right)^{-1}= & \left(\frac{C}{N}\right)_{\text {Feeder }}^{-1}+\left(\frac{C}{I}\right)_{\text {Feeder }}^{-1}+ \\
& \left(\frac{C}{I M}\right)^{-1}+\left(\frac{C}{N}\right)^{-1}+\left(\frac{C}{I}\right)^{-1} .
\end{aligned}
$$

$\left(\frac{C}{N}\right)$ and $\left(\frac{C}{I}\right)$ correspond to the user's signal to noise ratio and the user's signal to interference ratio. $\left(\frac{C}{N}\right)_{F e e d e r}$ and $\left(\frac{C}{I}\right)_{F e e d e r}$ are the feeder link's ${ }^{1}$ signal to noise ratio and feeder link's signal to interference ratio. $\left(\frac{C}{I M}\right)$ is the system's signal to intermodulation

\footnotetext{
${ }^{1}$ Feeder link is a communication link between the gateway and the satellite.
} 
product ratio. The terms $\left(\frac{C}{N}\right)_{F e e d e r},\left(\frac{C}{I}\right)_{F e e d e r}$ and $\left(\frac{C}{I M}\right)$ are constant in this study. Interference is more critical on the uplink where signals and interferences from the users are grouped together. According to this, only the frequency assignment problem on the uplink needs to be considered.

The $\left(\frac{C}{N}\right)$ for user $i$ is defined by

$$
\left(\frac{C}{N}\right)_{i}^{U p}=\frac{\left(E^{I R P T e r m} m_{i} / R S_{i}\right)}{L_{A t m o U p} \cdot L_{F S L U p}} \cdot \frac{G_{\text {Sat }\left(\text { Beam }_{i} \rightarrow i\right)}}{\left(T_{A}+T_{R e p}\right)} \cdot \frac{1}{k}
$$

EIRPTerm and $R S$ represent the Earth terminal's effective isotropic radiated power and the utilized symbol rate. $L_{A t m o U p}$ and $L_{F S L U p}$ are the uplink atmospheric loss and the uplink free space loss. $G_{S a t\left(B_{e a m} \rightarrow i\right)}$ corresponds to the antenna gain of the associated satellite beam at the user's position. The antenna and repeater equivalent noise temperature are denoted by $\left(T_{A}+T_{R e p}\right)$ while the Boltzmann constant is denoted by $k$.

The $\left(\frac{C}{I}\right)_{i}$ for user $i$ is defined by

$$
\begin{gathered}
\left(\frac{C}{I}\right)_{i}^{U p}=\frac{K_{i} \cdot G_{\text {Sat }\left(\text { Beam }_{i} \rightarrow i\right)}}{\sum_{j \in I n f} r_{i j} \cdot K_{j} \cdot G_{\text {Sat }\left(\text { Beam }_{i} \rightarrow j\right)}}, \\
K_{i}=\text { EIRPTerm }_{i} / R S_{i} \cdot L_{\text {AtmoUp }} \cdot L_{F S L U p},
\end{gathered}
$$

where $j \in \operatorname{Inf}$ refers to an interferer $j$ from a set Inf of interferers to the user $i$. Since users can have different frequency demands and interference level is dependant on the number of overlapping frequencies, an interference overlapping ratio $r_{i j}$ is defined such that $r_{i j}=o_{i j} / d_{i}$ where $o_{i j}$ denotes the number of overlapping frequencies between user $i$ and $j$.

Equations (1), (2) and (4) can then be rewritten in a linear form. By setting $A=\left(\frac{C}{N}\right)_{\text {Feeder }}^{-1}+\left(\frac{C}{I}\right)_{\text {Feeder }}^{-1}+\left(\frac{C}{I M}\right)^{-1}$, $B \quad=\left(\frac{C}{N}\right)_{i}^{-1}$,

$D$ $=\left(\frac{C}{N}\right)_{\text {Required }}$,

the Equation (1) can be expressed as

$$
\frac{1}{A+B+\frac{\sum_{j \in I n f} r_{i j} \cdot K_{j} \cdot G_{\text {Sat }\left(B e a m_{i} \rightarrow j\right)}}{K_{i} \cdot G_{\text {Sat }\left(\text { Beam }_{i} \rightarrow i\right)}}} \geq D
$$

and finally:

$$
\sum_{j \in I n f} r_{i j} \cdot \delta_{i j} \leq \alpha_{i}
$$

where

$$
\begin{aligned}
& \delta_{i j}=D \cdot K_{j} \cdot G_{\text {Sat }\left(\text { Beam }_{i} \rightarrow j\right)}, \\
& \alpha_{i}=K_{i} \cdot G_{\text {Sat }\left(\text { Beam }_{i} \rightarrow i\right)} \cdot(1-A D-B D) .
\end{aligned}
$$


If $\alpha_{i}$ is the acceptable interference threshold of user $i$ and $\delta_{i j}$ is the interference coefficient of user $j$ towards user $i$, the Equation (6) can be considered as the cumulative interference constraints for the user $i$.

It is now possible to provide a formulation of the FIAP. As explained in Section 1, scheduling models are designated.

\section{Scheduling models for the FIAP}

By considering a user as a task $i$ and its frequency demand $d_{i}$ as a processing time, maximizing the number of assigned users, each with contiguous frequencies according to a demand, based on a limited number of frequencies $B W$ is equivalent to minimizing the number of tardy tasks with a common due date $B W$. The minimization of the makespan (Cmax) problem which is a frequent problem in scheduling theory is also studied. The makespan minimization problem is linked to frequency assignment problem in that minimizing the makespan of a schedule gives the minimum greatest frequency used for a complete frequency assignment (all users are assigned). If all users may use all the bandwidth $\{0, \ldots, B W-1\}$, as it is the case in the problem studied here, this amounts to minimize the number of frequency used. Sections 3.1 and 3.2 propose, for the number of assigned users/number of tardy jobs criterion, an exact scheduling model and disjunctive approximations, respectively. Section 3.3 discusses the modifications of these models for the number of used frequencies/makespan criterion.

\subsection{The exact scheduling model with limited overlapping constraints}

Tasks can be overlapped and the overlap length should be taken into consideration in the constraints. Let $o_{i j}$ the overlap length between task $i$ and $j$. Minimizing the number of tardy tasks with a common due date $B W$ is given by:

$$
\begin{gathered}
\min \sum_{i=1}^{n} U_{i} \\
U_{i}= \begin{cases}1, & \text { if } f_{i}+d_{i} \geq B W, \\
0, & \text { otherwise }\end{cases}
\end{gathered}
$$

subject to

$$
\begin{gathered}
o_{i j}=\left|\left[f_{i}, f_{i}+d_{i}-1\right] \cap\left[f_{j}, f_{j}+d_{j}-1\right]\right| \quad \forall i, j \in\{1, \ldots, n\}, i \neq j \\
\sum_{j \in\{1, \ldots, n\} \backslash\{i\}} o_{i j} \delta_{i j} \leq d_{i} \alpha_{i} \quad \forall i \in\{1, \ldots, n\} \\
f_{i} \in \mathrm{N} \quad \forall i \in\{1, \ldots, n\}
\end{gathered}
$$


where variable $f_{i}$ denotes the starting time of the task $i$ and $d_{i}$ denotes task $i$ 's processing time. Equation (10) provides cumulative interference constraints and is similar to equation (6).

The general limited overlapping constraints given by (9-10) is new in scheduling. A related and well-studied constraints that limits the number of tasks that may overlap at a given time is given by the cumulative constraint, also called the discrete renewable resource constraint Baptiste et al. (2001). For classical cumulative or discrete resource constraints, there is a number $m$ of resources, each of capacity $B_{k}, k=1, \ldots, m$ and each task $i$ requires a non negative amount $b_{i k}$ of each resource $k$. The constraint specifies that, at any time, the total cumulated amount of each resource $k$ used by the activities in progress can not exceed $B_{k}$.

The following example illustrates the difference between the discrete renewable resource constraint and the limited overlapping constraint. Consider a three users/tasks example. A limited overlapping/cumulative interference constraint is defined for task $i=1$ for which $d_{1}=3, \alpha_{1}=2 / 3, \delta_{1 j}=1$ for $j=2,3$. The example also provides $d_{2}=1$ and $d_{3}=2$. Consider also a discrete renewable resource of capacity $B_{1}=2$, while $b_{11}=1, b_{21}=1$ and $b_{31}=1$. Four different schedules are displayed in Figure 1 .
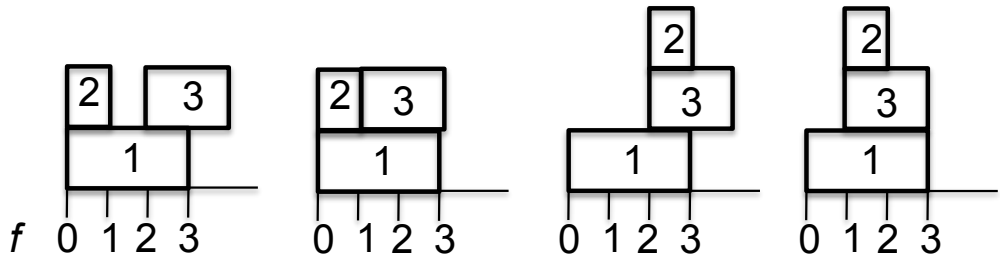

Figure 1. Different overlapping situations

For the considered numerical values the limited overlapping constraint can be written

$$
o_{12}+o_{13} \leq 2
$$

where $o_{12}$ denotes the overlapping length of tasks 1 and 2 while $o_{13}$ denotes the overlapping length of tasks 1 and 3. From left to right, schedules 1 and 3 satisfy the limited overlapping constraint while schedules 2 and 4 violate this constraint. The discrete resource constraint is satisfied for schedules 1 and 2 as each task uses a single unit of the resource and at each time the number of activities in process does not exceed 2 . However the discrete resource constraint is violated for schedules 3 and 4 at time $f=2$ for schedule 3 and $f=1$ for schedule 4 .

Such a limited overlapping constraint has not been considered in the scheduling literature. Note that the problem is NP hard as setting all $\alpha_{i}$ to 0 and some $\delta_{i j}$ to 1 reduces to the interval coloring problem or, equivalently, to the disjunctive scheduling problem. Finally, the limited overlapping constraint can be defined as a new extension of the disjunctive scheduling constraint.

In the absence of dedicated solution procedures, this paper proposes either to direct constraint programming modeling or to disjunctive scheduling approximations. For constraint programming, the IBM CPOptimizer constraint programming solver IBM ILOG, Inc (2012) which provides high level scheduling constraints and objects was used. Tasks can be considered as interval variables and the overlapping lengths $o_{i j}$ can be directly obtained by the function IloOverlapLength $(\mathrm{i}, \mathrm{j})$. The cumulative interference constraints $(9-10)$ can then be simply written as: 


$$
\sum_{j \neq i} \operatorname{IloOverlapLength~}(i, j) \delta_{i j} \leq d_{i} \alpha_{i} \quad \forall i \in\{1, \ldots, n\}
$$

\subsection{Disjunctive scheduling approximations}

Working on binary interference could help simplify the problem since it yields disjunctive scheduling constraints. Binary interferences are generated based on the existing cumulative interference environment. The presence of a binary interference between user $i$ and $j$ denoted by $\Delta_{i j} \in\{0,1\}$ is generated from interference coefficients $\delta_{i j}$ according to the following definition:

$$
\Delta_{i j}= \begin{cases}1, & \text { if } \delta_{i j} \geq L F \cdot \overline{\delta_{i j}} \vee \delta_{j i} \geq L F \cdot \overline{\delta_{i j}} \\ 0, & \text { otherwise }\end{cases}
$$

$L F \in[0,1]$ corresponds to a loading factor and $\overline{\delta_{i j}}=\frac{\sum \delta i j}{\sum k_{i j}}$ such that $k_{i j}=1$ if $\delta_{i j} \geq 0$ corresponds to the mean of all non-zero $\delta_{i j}$ values and 0 otherwise.

$L F$ determines how loaded the binary interference matrix is. If it is set to 1 , binary interference between user $i, j$ occurs if either one of their interference coefficients is not less than the mean $\overline{\delta_{i j}}$. The lower the $L F$ the denser the binary interference matrix is.

Note that $\delta_{i j} \neq \delta_{j i}$ since the present method considers the perceived interference at the satellite while distances between the satellite and the two users are different, but, for binary interference, it is necessary to set $\Delta_{i j}=\Delta_{j i}$. According to Equation (13), they are set to 1 if any of the $\left(\delta_{i j}, \delta_{j i}\right)$ pair is greater than the $L F \cdot \overline{\delta_{i j}}$ value.

Nonetheless, to ensure that the binary interference is a good estimation of the actual interference, the approach considered here needs to verify that each of the feasible solutions based on this binary interference constraints does not violate the actual cumulative interference constraints. In case of violation, $L F$ should be reduced in order to induce more binary interference relations.

Binary interference constraints between each couple of users can be treated as non-overlapping constraints between each couple of tasks. As already mentioned, the frequency interval assignment problem with binary interferences define a disjunctive scheduling problem without precedence constraints. A disjunctive scheduling problem is also commonly defined as a set of uninterrupted tasks with fixed durations that have to be performed on a set of machines while the machine can handle one task at a time Caseau and Laburthe (1995). The goal in this case is to order the tasks on the different machines according to the objective such as minimizing the total makespan of the schedule. Binary interference in the frequency assignment problem can be treated as disjunctive constraints in that two interfering users refer to two non-overlapping tasks. In non-interference case, tasks can be overlapped. As explained in the next section, this overlapping can be viewed as having tasks processed on different machines.

\subsubsection{Disjunctive graph and clique}

The problem can be represented by a disjunctive graph $G=(V, E)$ in which the vertices represent the users and an edge between two vertices represent their binary interference pair. Each edge of this disjunctive graph is treated as a disjunctive or non-overlapping constraint in scheduling.

The scheduling can be modeled by directly including each of these constraint pairs. Consider $n$ as a number of vertices, there are at most $n(n-1) / 2$ disjunctive constraints. 
Alternatively, it can be chosen to deal with a group of constraints using the maximal cliques concept.

A clique in a graph is defined as a subset of the vertices such that every two vertices in the subset are connected by an edge. A maximal clique is a clique that cannot be extended by including one more adjacent vertex.

A maximal clique in this case consists of a group of users that all interfere, i.e., in terms of scheduling, a machine shared by a group of tasks. By dealing with maximal cliques in the scheduling is similar to dealing with groups of constraints simultaneously which may lead to more efficient approaches. Among others, see e.g. Baptiste et al. (2001), the well known edge finding technique is able to detect implied precedence relations given the time windows of a set of tasks sharing the same machine.

The problem is that there may be an exponential number of maximal cliques given an arbitrary binary interference graph.

Below is an example of a disjunctive graph with $\{1,2\},\{2,3\},\{2,4\},\{3,5\}$ and $\{4,5,6\}$ as its maximal cliques.

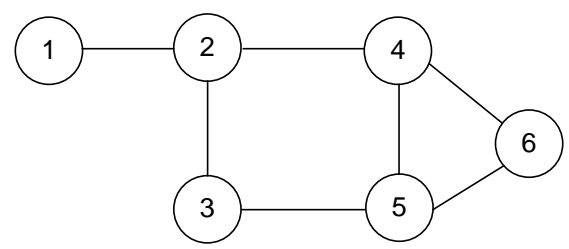

Figure 2. A disjunctive graph

A clique can be viewed as a machine in disjunctive scheduling. In this example, there are 5 machines $m_{1}, \ldots, m_{5}$. These machines can be represented by a binary matrix with rows for machines $m_{1}, \ldots, m_{5}$ and columns for tasks $1, \ldots, 6$ as

$$
\left[\begin{array}{llllll}
1 & 1 & 0 & 0 & 0 & 0 \\
0 & 1 & 1 & 0 & 0 & 0 \\
0 & 1 & 0 & 1 & 0 & 0 \\
0 & 0 & 1 & 0 & 1 & 0 \\
0 & 0 & 0 & 1 & 1 & 1
\end{array}\right] .
$$

Tasks associated to the same machine cannot be overlapped. According to this disjunctive graph, if the disjunctive constraints are modeled directly, 7 pairs of constraints are needed. If maximal clique concept is used, the constraints can be modeled based on 5 cliques.

\subsubsection{Disjunctive scheduling models}

By considering a user as a task $i$ and its frequency demand $d_{i}$ as a processing time, the equivalent $n$ tasks scheduling problem can be modeled as follows:

$$
\min \sum_{i=1}^{n} U_{i}
$$

subject to 


$$
\begin{gathered}
U_{i}=\left\{\begin{array}{ll}
1, & \text { if } f_{i}+d_{i} \geq B W, \\
0, & \text { otherwise, }
\end{array} \quad \forall i \in\{1, \ldots, n\}\right. \\
f_{i}-f_{j} \geq d_{j} \vee f_{j}-f_{i} \geq d_{i}, \quad \forall i, j \in I, i \neq j,
\end{gathered}
$$

and domain constraints (11).

Set $I$ contains all the pairs of tasks that are independent (non-overlapping) to one another. These pairs correspond to the pairs of vertices of the disjunctive graph mentioned above.

In case of cliques, set $I$ is replaced with a number of sets which each contains tasks that are all independent. Constraint (16) becomes

$$
f_{i}-f_{j} \geq d_{j} \vee f_{j}-f_{i} \geq d_{i} \quad \forall i, j \in C, i \neq j, \forall C \in \mathcal{C}
$$

where $\mathcal{C}$ is the set of all considered cliques.

Equations (16) and (17) are equivalent provided that $\mathcal{C}$ is a set of cliques of the disjunctive graph such that each disjunctive constraint is included in a clique of $\mathcal{C}$. This holds in particular if $\mathcal{C}$ is the set of maximal cliques.

This scheduling problem is equivalent to the FAP with maximization of the number of served users with no interference allowed. Remark that when the interference graph is complete, there is a single maximal clique, i.e. a single machine. The corresponding scheduling problem is denoted $1\left|D_{i}=D\right| \sum U_{i}$ and is simply solved by sorting the tasks with the SPT rule (shortest processing time) and by scheduling the maximum number of tasks before $D$ in that order ${ }^{1}$. For the general case, the problem is NP-hard as it includes for instance the job-shop problem.

Remark 1 Note this model could also consider non necessary contiguous frequencies demand by duplicating each user node into several nodes (one node per frequency demand) and adapting the interference graph accordingly.

Remark 2 More general interferences of the form $\left|f_{j}-f_{i}\right| \geq \Delta_{i j}$ could be tackled by considering setup times on the machine corresponding to the clique.

\subsection{Minimizing the maximum frequency used}

In this section, another criterion is considered. The objective is to find an assignment for each user (no rejected user) regarding his demand which minimizes the greatest frequency used. To compare with scheduling problems, the approach of thid paper corresponds to a disjunctive scheduling problem with minimization of the completion time of the last job called makespan and denoted $C_{\max }$.

It leads to the following model for $n$ users.

$$
\min C_{\max }=B W
$$

\footnotetext{
${ }^{1}$ The notation $D_{i}$ is used instead of $d_{i}$ which is generally encountered in scheduling since in this article $d_{i}$ stands for the user demand.
} 
such that

$$
B W \geq f_{i}+d_{i} \quad \forall i \in\{1, \ldots, n\} .
$$

Interference should also be took into account in this model. For the exact CP model, constraints (11-12) are added. For the disjunctive scheduling approximations, as in §3.2.2, either pairs of tasks (equation (16)) or maximal cliques (equation (17)) in addition to constraints (11) are considered in both cases.

\section{Computational experiments}

All the models are coded in $\mathrm{C}++$ and solved using IBM CP Optimizer IBM ILOG, Inc (2012). The results were obtained on a $2.7 \mathrm{GHz}$ Intel Core i5 machine with 4GB RAM. The CPU times for the calculations were limited to 60 seconds, unless stated otherwise.

The test instances are extracted from an industrial application of FAP for SDMA satellite communication systems provided by Thales Alenia Space, the complete description is available in Kiatmanaroj et al. (2012).

From this application, single carrier FAP problems were previously generated and solved in Houssin et al. (2011) and K.Kiatmanaroj et al. (2013). The only difference from the instances considered in these papers is that multiple consecutive frequencies (intervals) are required. The user positions are randomly generated and uniformly distributed over the service area defined by a set of geographic coordinates $u=[-0.043980,0.048520]$ and $v=[-0.021152,0.012348]$. For each instance and for each user, the frequency demand belongs to $\{5,10,15,20\} \mathrm{MHz}$. The bandwidth parameter range (when fixed) will be specified in what follows. The instances and the parameters used to compute the cumulative interferences are provided in homepages.laas.fr/lhoussin/FAP/SDMA_Sat_FAP.htm.

First, the effect of considering maximum cliques instead of constraint pairs on binary interference FAP is quantified. Then, the paper validates the transformation of a cumulative FAP to a binary FAP by checking constraint violations for several parameters of the conversion. Finally, the complete method (binary transformation and maximum cliques) is applied to a set of cumulative problems.

\subsection{Effect of considering maximum cliques}

Experiments start by studying the benefit of using maximal cliques vs the interference matrix model for the disjunctive approximation of the problem. The question is to evaluate the benefit of adding maximal cliques and also if adding too many cliques may slow down the $\mathrm{CP}$ solver or not for the considered industrial application.

\subsubsection{Test on artificially large instances}

The minimum makespan problem over 100 instances of 500 and 1,000 users was solved by varying the maximal cliques usage in the model from $100 \%$ (using all maximal cliques) to $0 \%$ (using all constraint pairs). The number of optima was also counted. Note that maximal cliques were enumerated using Bron-Kerbosch algorithm Bron and Kerbosch (1973), Pardalos and Xue (1994), Cazals and Karande (2008) which is Algorithm 457 in ACM collection. The algorithm is also coded in $\mathrm{C}++$.

The results are shown in Figure 3. It can be seen that considering all maximal cliques always help improving the performance of the calculation. Makespan is considerably lower in 100\% maximal cliques usage compared to none. The trend is similar even for the 
1,000 user case which is considered hard to solve and far larger than realistic instances (see next Section).

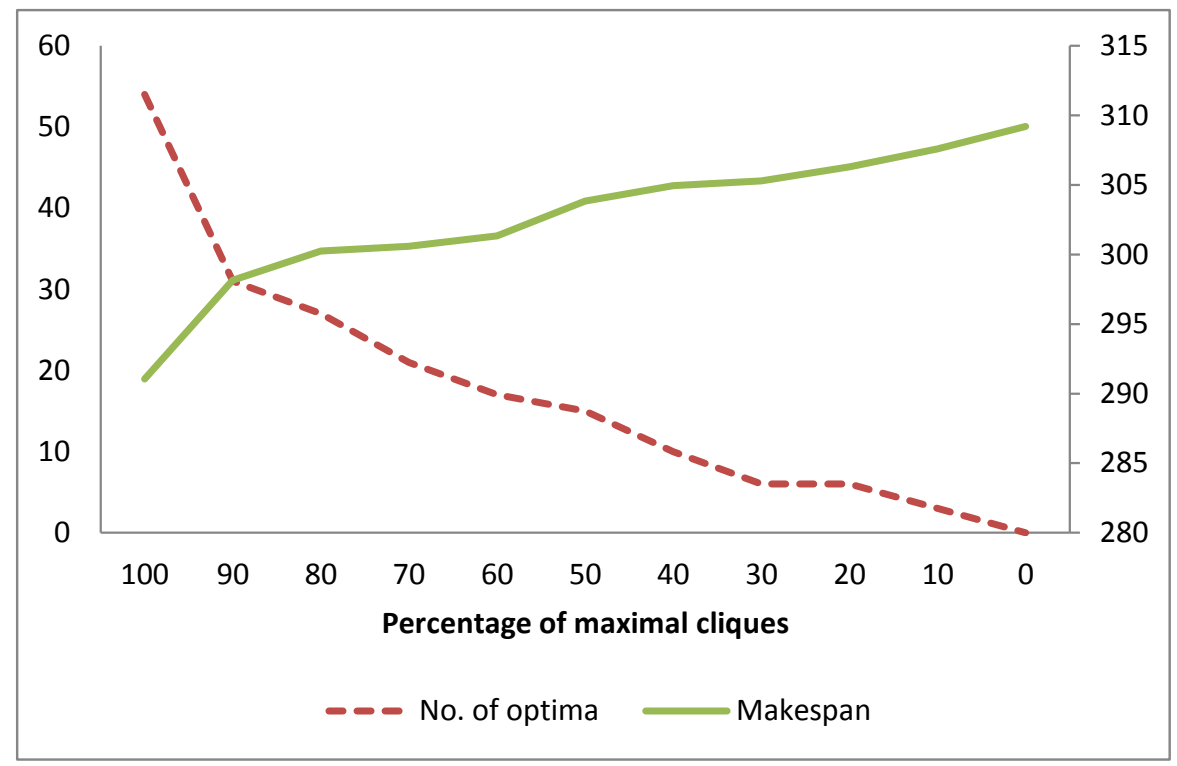

(a)

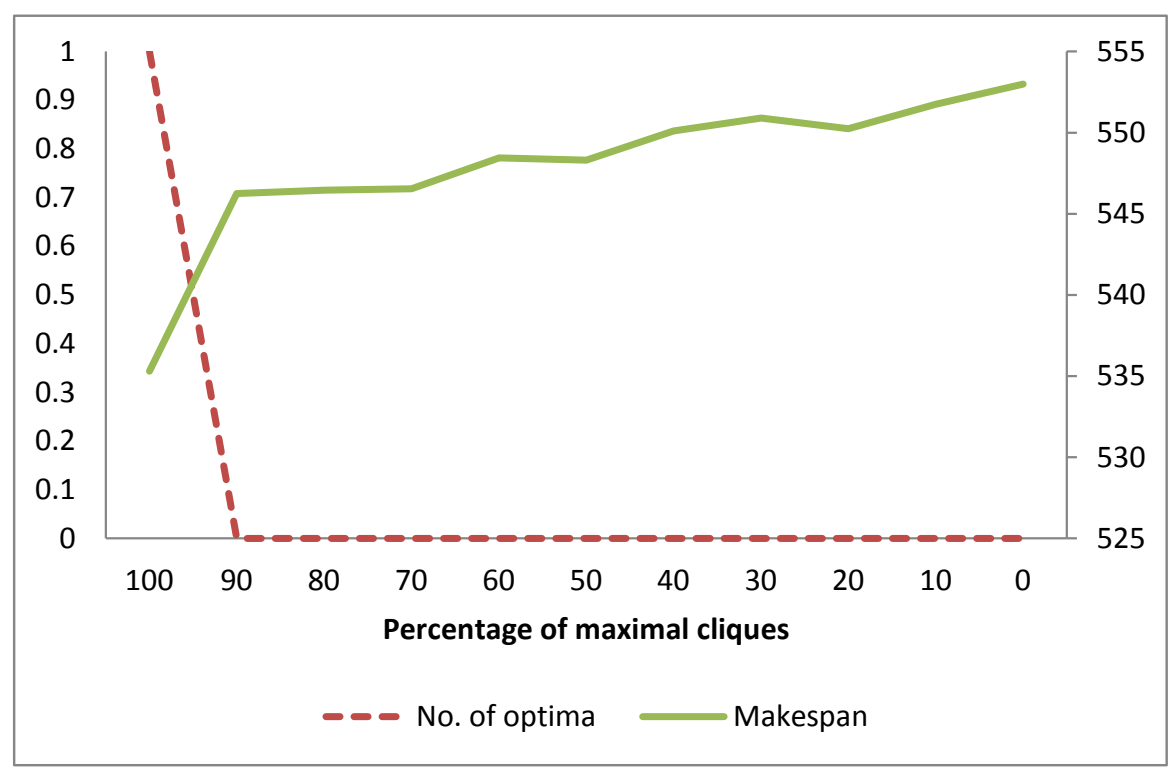

(b)

Figure 3. Number of optima and makespan versus percentage of maximal clique usage (a) 500 users (b) 1000 users

Average numbers of maximal cliques and the average time needed to list all of them are shown in Table 1. The maximal clique listing time is low for 500 users but rather high for 1,000 users. Note that, for a large number of users, a faster depth-first search algorithm Tomita et al. (2006) which employs the same pruning method as Bron-Kerbosh algorithm could be used.

The conclusion of this section is that for the used realistic FIAP instances solved by disjunctive approximation and for the makespan criterion, the $\mathrm{CP}$ solver is never penalized by the adjunction of all maximal cliques even when their number if very large. 
Table 1. Maximal cliques computation performances

\begin{tabular}{lrr}
\hline & 500 users & 1,000 users \\
\hline Average number of maximal cliques & 1135.2 & 5241.52 \\
Average time to list maximal cliques (s.) & 1.03 & 22.95 \\
\hline
\end{tabular}

Table 2. CP Optimizer solver status at $60 \mathrm{MHz}$ bandwidth and 60 seconds solving time

\begin{tabular}{lrrrrr}
\hline Instances & \# branches & \# fails & \# Choice points & \# variables & \# constraints \\
\hline MC_1 & 61464 & 19831 & 42707 & 2050 & 3501 \\
MC_2 & 65981 & 24436 & 42225 & 2167 & 3735 \\
MC_3 & 53513 & 19140 & 34592 & 2563 & 4527 \\
IM_1 & 107787 & 38501 & 71892 & 2781 & 4963 \\
IM_2 & 120988 & 43058 & 80548 & 2959 & 5319 \\
IM_3 & 109413 & 38081 & 74319 & 3001 & 5403 \\
\hline
\end{tabular}

Can the same trend be observed for the maximal number of served users objective?

\subsubsection{Makespan and number of assigned users on realistic instances}

Smaller, more realistic, instances for both the makesan/bandwidth criterion and the number of assigned users criterion are now considered, increasing stepwise the number of users by 20 from 20 to 200 users with 100 instances each.

For minimizing makespan problem, a test comparing the results when using constraints from maximal cliques (MC) and constraints directly from binary interference matrix (IM) was conducted. Results are shown in Figure 4. As expected, the model utilizing maximal cliques gives better results both in terms of makespan and the number of optima found. Nonetheless, the gap is small for makespan.

For the maximum number of assigned users, the performance gap is also small with a common due date (bandwidth) set to $60 \mathrm{MHz}$ or to $100 \mathrm{MHz}$, see Figure 5. Both models show the consistent trends when the frequency resource is increased from $60 \mathrm{MHz}$ to 100 MHz. The number of optima gap between both models is the same in $60 \mathrm{MHz}$ and 100 $\mathrm{MHz}$ bandwidth. In this case however, maximal cliques gives slightly worse results in term of the number of assigned users. But in terms of number of optima, it still performs better. Nonetheless, when looking into more details on the solver's parameter shown in Table 2, the models with constraints based directly on interference matrix (IM_1 to IM_3) yields almost twice the number of searching branches, requires higher number of variables and constraints. Thus, considering a longer solving time, the models with constraints based on maximal cliques (MC_1 to MC_3) have more potential to give better results. In what follows, disjunctive approximations are always solved after incorporating all maximal cliques.

\subsection{Performance of disjunctive scheduling approximations}

In order to obtain further insights on the disjunctive approximation, the approach considered here needs to find good binary interference matrices to base the comparison on. For this, the present method has to check if each solution found for the disjunctive approximation is feasible for the limited overlapping constraints. Such experiments are presented below for the makespan criterion.

By varying the loading factor values, the total number of constraint violations and number of optima are provided in Tables 3 and 4. Reducing the LF causes more interference load (higher number of interference pairs) resulting in harder instances to solve as can be seen from reduction of the number of optima. However, the number of limited overlapping constraint violations decreases. As a result of these experiments, the inter- 


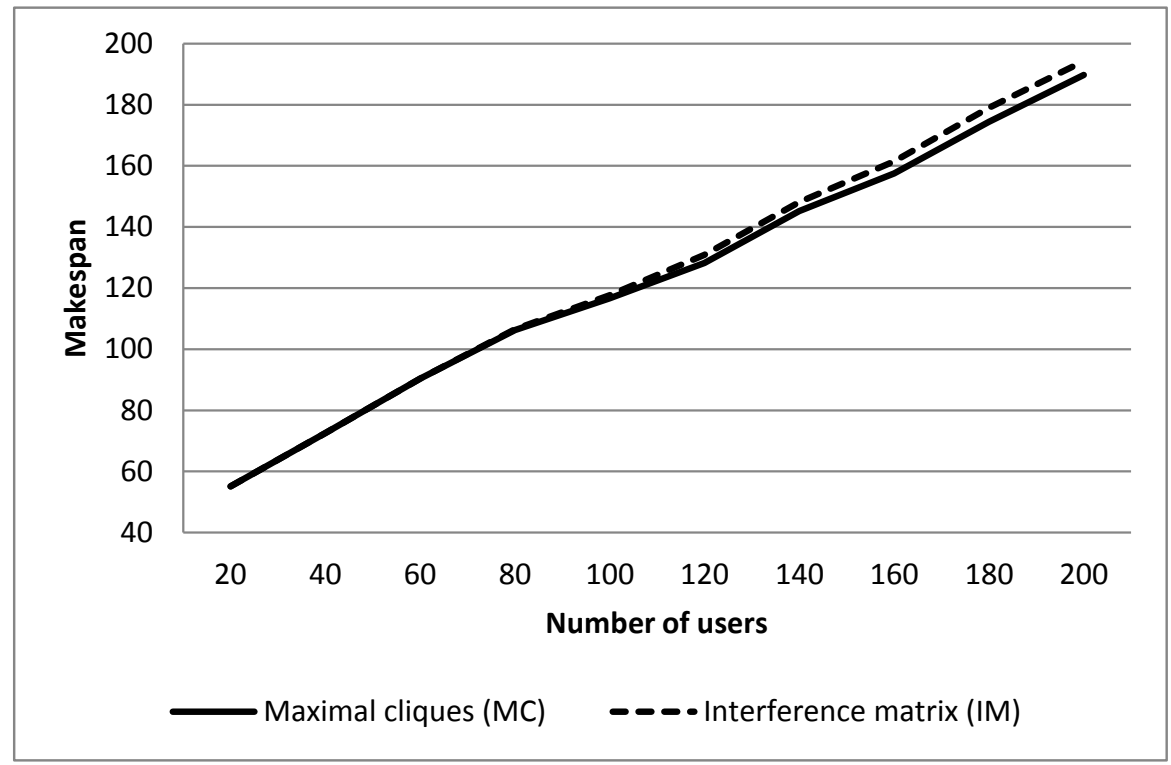

(a)

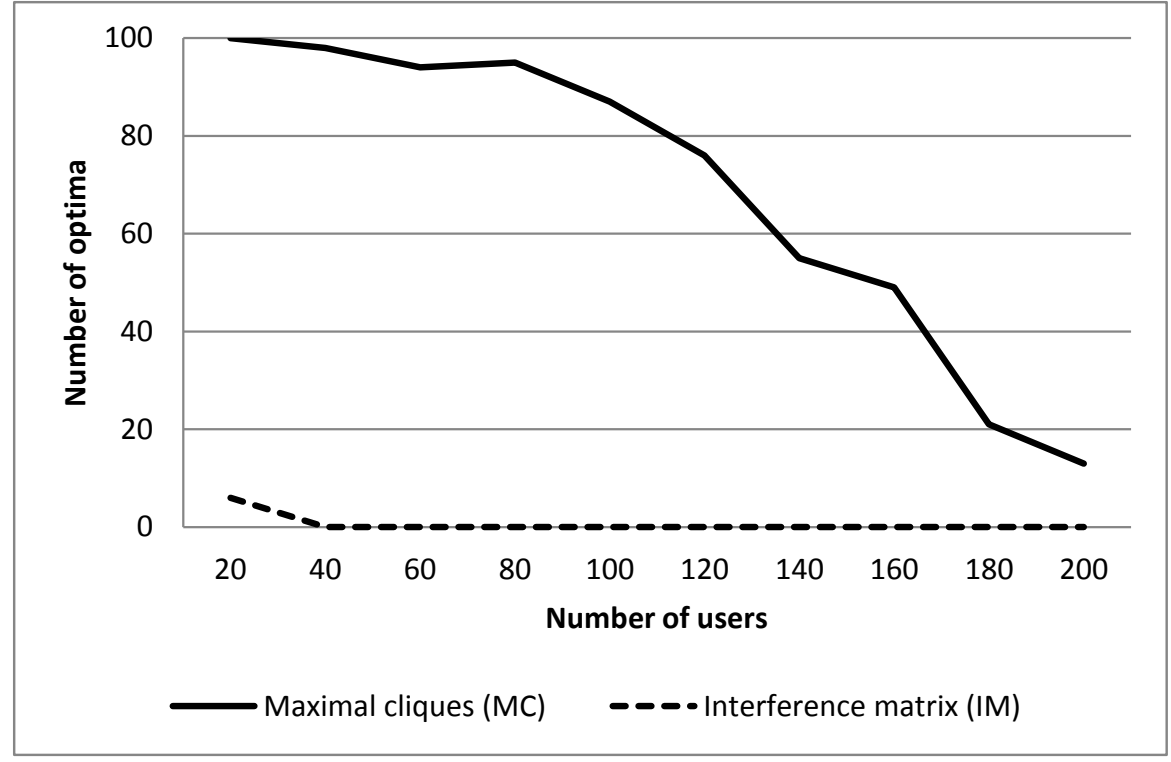

(b)

Figure 4. Makespan and number of optima by using constraints either from maximal cliques or binary interference matrix

Table 3. Number of constraint violations for different values of loading factors

\begin{tabular}{lrrrrrrrrrr}
\hline Number of users & 20 & 40 & 60 & 80 & 100 & 120 & 140 & 160 & 180 & 200 \\
\hline 0.8 & 11 & 103 & 329 & 753 & 1063 & 1589 & 2007 & 2793 & 3170 & 4161 \\
0.6 & 3 & 13 & 15 & 52 & 54 & 69 & 137 & 168 & 197 & 290 \\
0.5 & 2 & 1 & 3 & 3 & 3 & 5 & 5 & 12 & 7 & 19 \\
0.4 & 2 & 0 & 0 & 0 & 0 & 0 & 0 & 0 & 0 & 0 \\
\hline
\end{tabular}

ference matrices based on the loading factor 0.4 (no constraint violation for more than 40 users and only 2 violations for the 100 instances of 20 users) are selected for further tests.

It is now possible to compare the best disjunctive approximation with the direct solving 


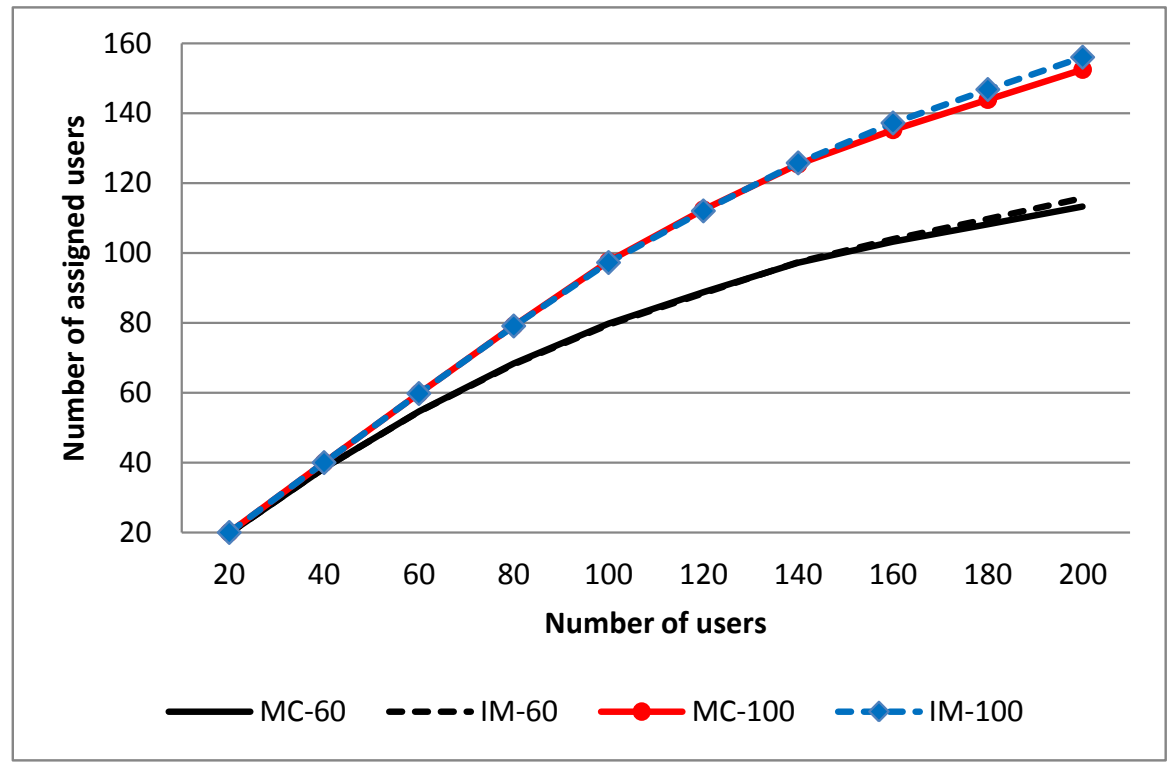

(a)

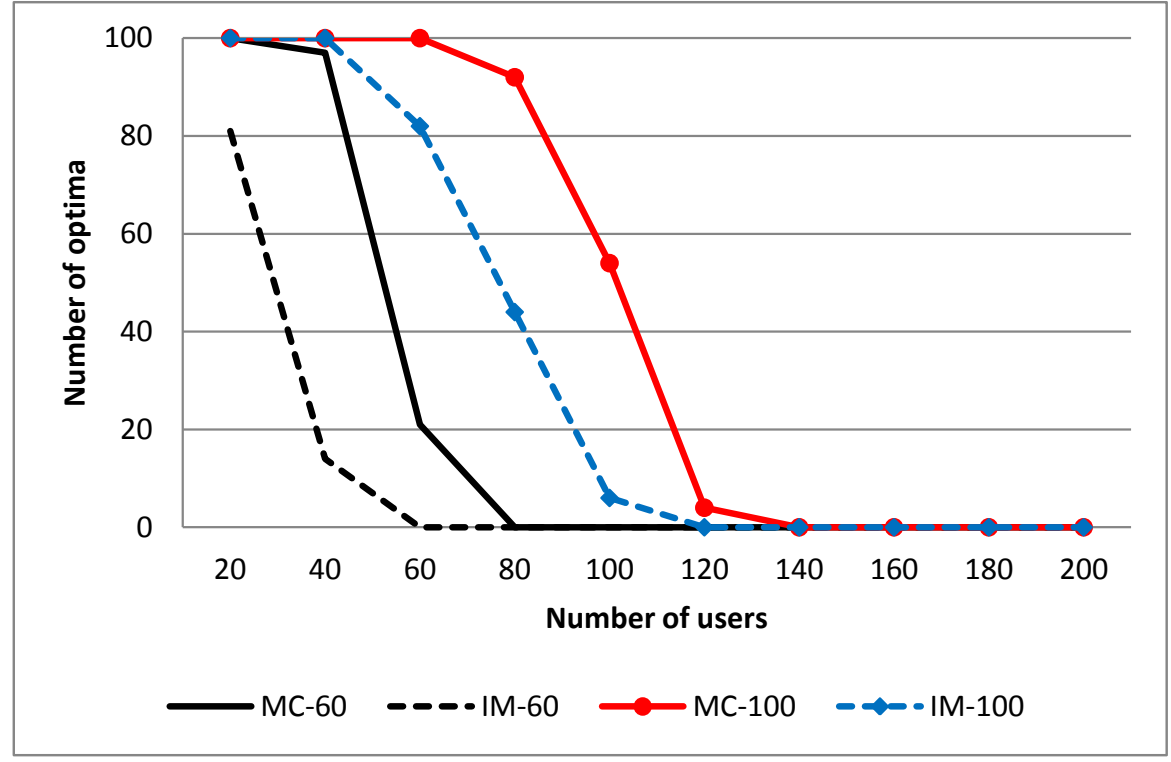

(b)

Figure 5. Number of assigned users by using constraints either from maximal cliques (MC) or binary interference matrix (IM)

Table 4. Number of optima for different values of loading factors

\begin{tabular}{lrrrrrrrrrr}
\hline Number of users & 20 & 40 & 60 & 80 & 100 & 120 & 140 & 160 & 180 & 200 \\
\hline 0.8 & 100 & 100 & 100 & 100 & 99 & 97 & 97 & 99 & 96 & 96 \\
0.6 & 100 & 95 & 98 & 95 & 93 & 84 & 77 & 66 & 46 & 34 \\
0.5 & 100 & 98 & 94 & 95 & 87 & 76 & 55 & 49 & 21 & 13 \\
0.4 & 100 & 97 & 95 & 92 & 82 & 64 & 47 & 21 & 10 & 5 \\
\hline
\end{tabular}

of the scheduling problem with limited overlapping constraint. For the number of assigned users criterion, similarly to the binary interference case, two bandwidth settings are used: $60 \mathrm{MHz}$ and $100 \mathrm{MHz}$. Results are compared with the corresponding binary case with different loading factors, see Figure 6. 


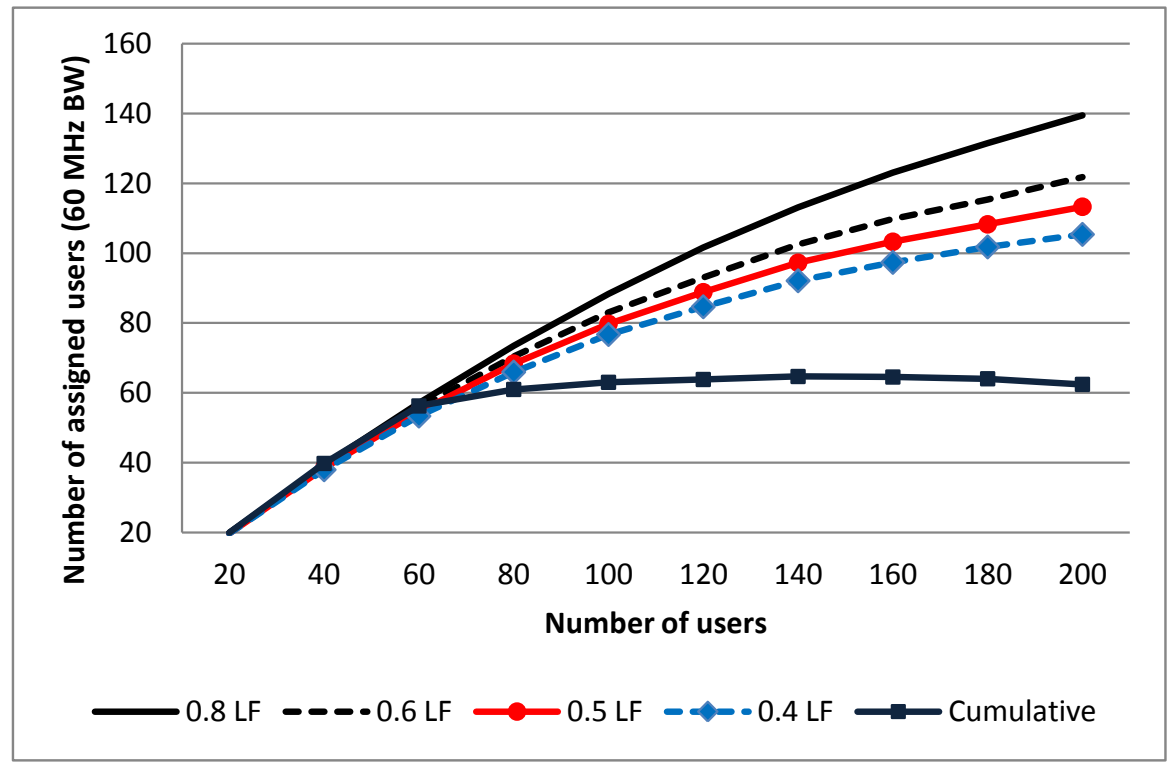

(a)

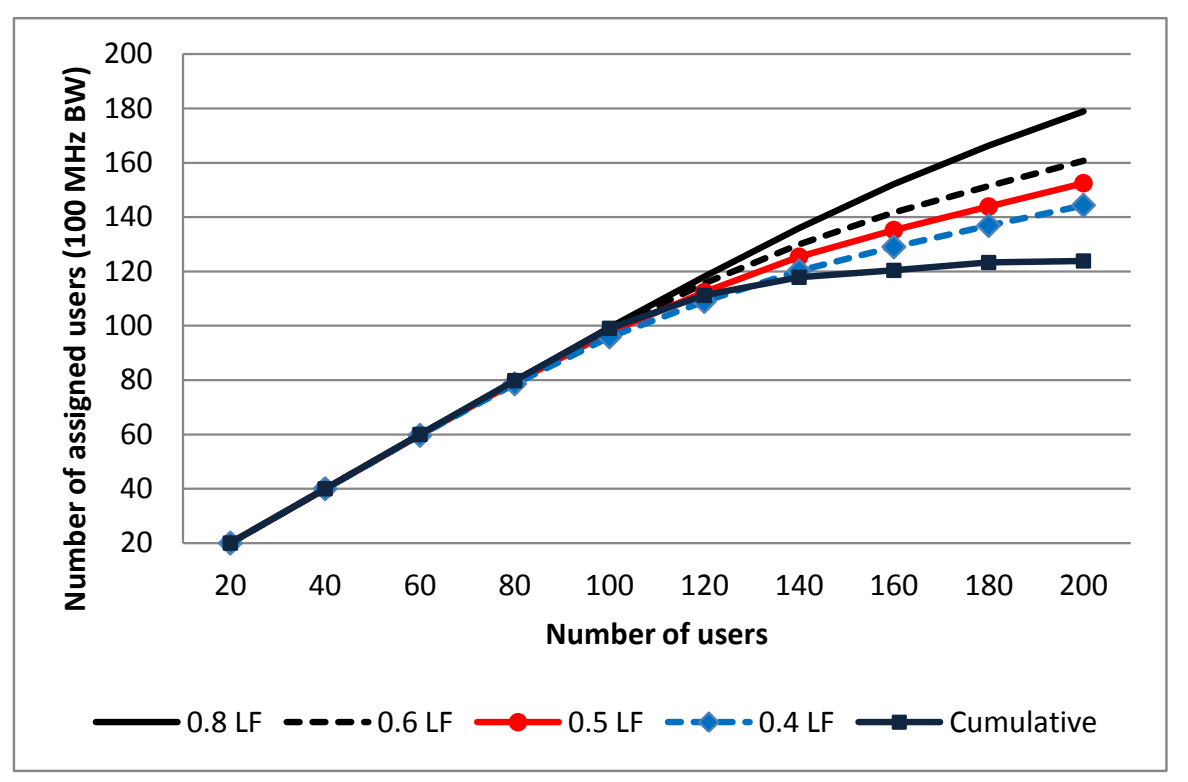

(b)

Figure 6. Comparison between the cumulative interference and binary interference with different loading factors in term of number of assigned users (a) $60 \mathrm{MHz}$ bandwidth (b) $100 \mathrm{MHz}$ bandwidth

\begin{abstract}
The number of assigned users saturate at 60 users for $60 \mathrm{MHz}$ bandwidth case and 120 users for $100 \mathrm{MHz}$ bandwidth case. Both are much lower than the results based on binary interference even that with loading factor of 0.4 with only two constraint violations over 1000 instances. This poses an interesting finding in that solving the industrial application with the binary interference constructed from the actual interference matrix is easier and gives better results. This finding is consistent with the experiments made by Graham et al. Graham et al. (2008) in a different FAP context.
\end{abstract}

.




\section{Conclusions}

In this paper it is shown that frequency interval assignment, even with cumulative interferences, can be treated as a scheduling problem which can be solved efficiently, at least for the considered industrial application.

The technique that aims to construct a binary interference problem from a cumulative interference one is simple and, for a good tuning of parameters, Table 3 shows that the method is efficient on the tested instance set. Moreover, two scheduling models are proposed, the first one consider constraints on pairs of disjunctive tasks and the second one involves maximal cliques. Despite the difficulty to find all maximal cliques in a graph, numerical experiments show that the performance is mostly improved when a large number of maximal cliques is computed.

The performance is improved further when the cumulative interference is transformed to binary interference and solve the problem using the combined scheduling-based formulation and maximal clique concept.

It should finally be stressed that the solution should be verified in that it should not cause constraint violation based on the cumulative interference. It was almost not the case in the industrial application except for small 20 users instances that were easy to solve, even by the direct model. For other applications, repairing methods could be of interest for obtaining a feasible solution from the disjunctive approximation. This could be the basis of further research. Finally, this paper has exhibited a new scheduling problem with limited overlapping constraints, that could be studied specifically as it constitutes a challenging extension of the disjunctive scheduling problem.

\section{References}

Aardal, K., van Hoesel, S.P.M., Koster, A.M.C.A., Mannino, C., Sassano, A., 2007. Models and solution techniques for frequency assignment problems. Annals OR 153,1, 79-129.

Baptiste, P., Pape, C.L., Nuijten, W., 2001. Constraint-Based Scheduling. Springer US.

Bouchard, M., Cangalovic, M., Hertz, A., 2010. On a reduction of the interval coloring problem to a series of bandwidth coloring problems. Journal of Scheduling 13, 583-595.

Bron, C., Kerbosch, J., 1973. Algorithm 457: finding all cliques of an undirected graph. Commun. ACM 16, 575-577.

Caseau, Y., Laburthe, F., 1995. Disjunctive scheduling with task intervals, Laboratoire dInformatique de lEcole Normale Suprieure. Technical Report 95-25.

Cazals, F., Karande, C., 2008. A note on the problem of reporting maximal cliques. Theoretical Computer Science Elsevier. 407, $564-568$.

Corbel, E., juillet 2008. Gestion de la ressource radio dans un système utilisant le SDMA. Technical Report RT2E-TAS-MO-641. THALES Alenia Space.

Dorndorf, U., Pesch, E., n Phan-Huy, T., 2000. Constraint propagation techniques for the disjunctive scheduling problem. Artificial Intelligence 122, $189-240$.

Graham, J.S., Montemanni, R., Moon, J.N.J., Smith, D.H., 2008. Frequency assignment, multiple interference and binary constraints. Wirel. Netw. 14, 449-464.

Houssin, L., Artigues, C., Corbel, E., 2011. Frequency allocation problem in a sdma satellite communication system. Computers \& Industrial Engineering 61, 346 - 351.

IBM ILOG, Inc, 2012. IBM ILOG CPLEX: High-performance software for mathematical programming and optimization. See http://www-01.ibm.com/software/websphere/ilog/.

Kiatmanaroj, K., Artigues, C., Houssin, L., Corbel, E., 2012. Greedy algorithms for timefrequency allocation in a sdma satellite communication system, in: 9th International Conference on Modeling, Optimization \& SIMulation (MOSIM 2012), Bordeaux. URL: http: //hal.archives-ouvertes.fr/docs/00/72/86/45/PDF/paper_215.pdf.

K.Kiatmanaroj, C.Artigues, L.Houssin, F.Messine, 2013. Frequency assignment in a sdma satel- 
lite communication system with beam decentring feature. Computational Optimization and Applications. 56.2.

Mannino, C., Sassano, A., 2003. An enumerative algorithm for the frequency assignment problem. Discrete Applied Mathematics 129, 155-169.

Metzger, B.H., 1970. Spectrum management technique. Presentation at 38th National ORSA meeting (Detroit, MI).

Montemanni, R., Smith, D.H., 2010. Heuristic manipulation, tabu search and frequency assignment. Computers \& Operations Research 37.

Muñoz, D.F., Muñoz, D.F., 2012. Algorithms for the generalized weighted frequency assignment problem. Computers \& Operations Research 39.

Palpant, M., Oliva, C., Artigues, C., Michelon, P., Biha, M.D., 2008. Models and methods for frequency allocation with cumulative interference constraints. International Transactions in Operational Research 15, 307-324.

Pardalos, P.M., Xue, J., 1994. The maximum clique problem. Journal of global Optimization 4, 301-328.

Pinedo, M.L., 2012. Scheduling: Theory, Algorithms, and Systems. 4th ed., Springer Publishing Company, Incorporated.

Tomita, E., Tanaka, A., Takahashi, H., 2006. The worst-case time complexity for generating all maximal cliques and computational experiments. Theor. Comput. Sci. 363, 28-42.

de Werra, D., Gay, Y., 1994. Chromatic scheduling and frequency assignment. Discrete Applied Mathematics 49, 165 - 174. 